matrix. Fracture tests were performed using double-cantilever-beam specimens. Environmental scanning electron micrographs showed a thin polymer film on the fracture surface and illustrated the rupture process of an embedded microcapsule. Infrared spectroscopy supplied chemical information confirming that the film is the polymerized healing agent. Virgin and healed load-displacement curves from fracture tests assessed the crack-healing efficiency of the composite sample, which in this case was up to $75 \%$ recovery of the virgin fracture toughness.

"Self-healing composites possess great potential for solving some of the most limiting problems of polymeric structural materials: microcracking and hidden damage," said the researchers. They said that the material cannot only "recover structural function," but also "increase the reliability and service life of thermosetting polymers used in a wide variety of applications ranging from microelectronics to aerospace."

\section{Increased Lateral Electrostriction Realized in Ferroelectric Liquid- Crystalline Elastomer Films}

The strain due to the external electric field of electrostrictive materials forms the basis of such micromachines as transducers, actuators, pumps, and medical microrobots. Of the reported electrostrictive materials-including piezoelectric crystals, grafted polyglutamates, carbon nanotubes, and polycopolymers-the best performance exhibited in these materials is $4 \%$ strain at an electric field $150 \mathrm{MVm}^{-1}$. Ferroelectric liquid-crystalline elastomer (FLCE) films, however, have shown the same constriction of $4 \%$, but require only a $100 \times$ lower electric field of $1.5 \mathrm{MVm}^{-1}$.

F. Kremer, whose group studied the films at the Institute for Experimental Physics at Leipzig University, said the principle of FLCE lies in the fact that the orientation of liquid crystal (LC) molecules is influenced by an applied electric field, and that the ferroelectric LC molecules can respond to the external electric field at a very high speed.

As reported in the March 22 issue of Nature, the paraelectric $S_{A}{ }^{*}$ phase LC material organizes itself in a layered liquid-crystalline structure. In each layer, the long axes of the rodlike LC molecules are aligned perpendicular to the layer plane. Such LC polymers can be synthesized by means of attaching the LC molecules to a polysiloxane backbone perpendicularly with a flexible alkyl chain, and cross-linking of the polymer LC. In this way, the layered structure can be preserved under mechanical stress. When a lateral electric field is applied, the LC molecules will tilt at an angle proportional to the external electric field, thus thinning the layer and the overall thickness of the film.

Mathematical analysis shows that the constriction of FLCE films is proportional to the square of the lateral electric field, and that the frequency of the effect is twice that of the applied alternating electric field, which is characteristic of electrostriction. The electrostriction of a freely suspended FLCE film was measured at continuously increasing voltages. The square dependence of the electrostriction on the external lateral electric field could be clearly seen. The electrostriction coefficient of the FLCE was also measured at different temperatures, demonstrating that the electrostriction coefficient of FLCE films varies with temperature. The maximum electrostriction coefficient was found to appear near the Curie temperature at which the phase transition of the LC material from the ferroelectric $\left(S_{C}{ }^{*}\right)$ to paraelectric $S_{\mathrm{A}}{ }^{*}$ phase occurs.

The researchers expect the FLCEs high mechanical response to an electrical field to be useful in nanomachine applications. SHUYAN ZHANG

\section{Low-Temperature Synthesis of Stable, Insoluble Uranium Boride Demonstrated}

Storage of plutonium presents a myriad of problems from pollution concerns to nuclear weapon proliferation risk. It has long been known that plutonium and boron could be combined to create a very stable and insoluble compound, plutonium boride. However, this could only be done at temperatures higher than $3000^{\circ} \mathrm{C}$. The two elements are melted at a very high temperature, cooled, ground into a powder, then mixed and melted again. Sometimes this process must be done several times in order to achieve proper mixing. Kent Abney of Isotope and Nuclear Chemistry (C-INC), along with Anthony Lupinetti, a postdoc with a dual CDivision and Nuclear Materials Technology (NMT) Division appointment, and Ed Garcia also from NMT, have developed a reactive process that occurs at more easily attainable temperatures, between $400^{\circ} \mathrm{C}$ and $800^{\circ} \mathrm{C}$, and does not involve repeating the process. While the researchers used uranium, the implication is that the process will work similarly for plutonium.

Lupinetti said, "By combining actinide metal halides, like uranium tetra- and trichlorides with molecular boron precursors like magnesium diboride or calcium hexaboride, we've been able to do reactions at temperatures in the $500-800^{\circ} \mathrm{C}$ range."

As the researchers reported at the 221st American Chemical Society National
Meeting in San Diego in April, they studied the reactions taking place in a small sealed quartz tube. The tube, under vacuum to remove all gasses and water vapor, was heated in a small electric furnace over a period of 1-5 days with a three-day cool down. The resultant compounds were later analyzed through $\mathrm{x}$-ray powder diffraction.

The researchers found that the end result of a uranium tetra-chloride reaction with magnesium diboride yields uranium boride mixed with a magnesium chloride. The latter is easily washed away, leaving behind the uranium-boride, a compound that is stable and insoluble. In addition, actinides mixed with boron, which readily absorbs neutrons, are not easily converted to their pure form, making them harder to use in weapons.

\section{Spectroscopic Technique Reveals Motion of Single Molecules Within a Medium}

Using single-molecule spectroscopy, researchers at the University of TexasAustin have discovered a method for observing the movement of individual molecules as well as insights into molecular motion in supercooled liquids. As reported in the April 13 issue of Science, graduate student Laura A. Deschenes and assistant professor David A. Vanden Bout, of the Department of Chemistry and Biochemistry, made refinements in previously used laser technology to isolate and illuminate a molecule called rhodamine 6G. Rhodamine 6G is a strongly fluorescent molecule used as the lasing medium in a yellow/orange/red dye laser. Because the method allowed molecules to be observed individually, the researchers were able to avoid the jumbling effect that masks what is really happening.

"If you look at all the molecules at the same time, you can't tell the difference in their movements. You get a jumble of times," Vanden Bout said. "What we discovered is that the rotations of individual molecules are all very different from each other. Some of the molecules are moving really fast, and some are really slow. In our supercooled liquid, there are regions within the liquid that are different from one another."

With single-molecule spectroscopy, the researchers were able to measure the dynamics of any region where a probe molecule is found. They found that the domains move at different rates from one another, and within each domain, the motion appeared as the motion of normal liquid.

Vanden Bout said they also discovered that "the domains are not static. We can watch them interchange with one another. When a fast-moving molecule switches environments, it becomes slow." 